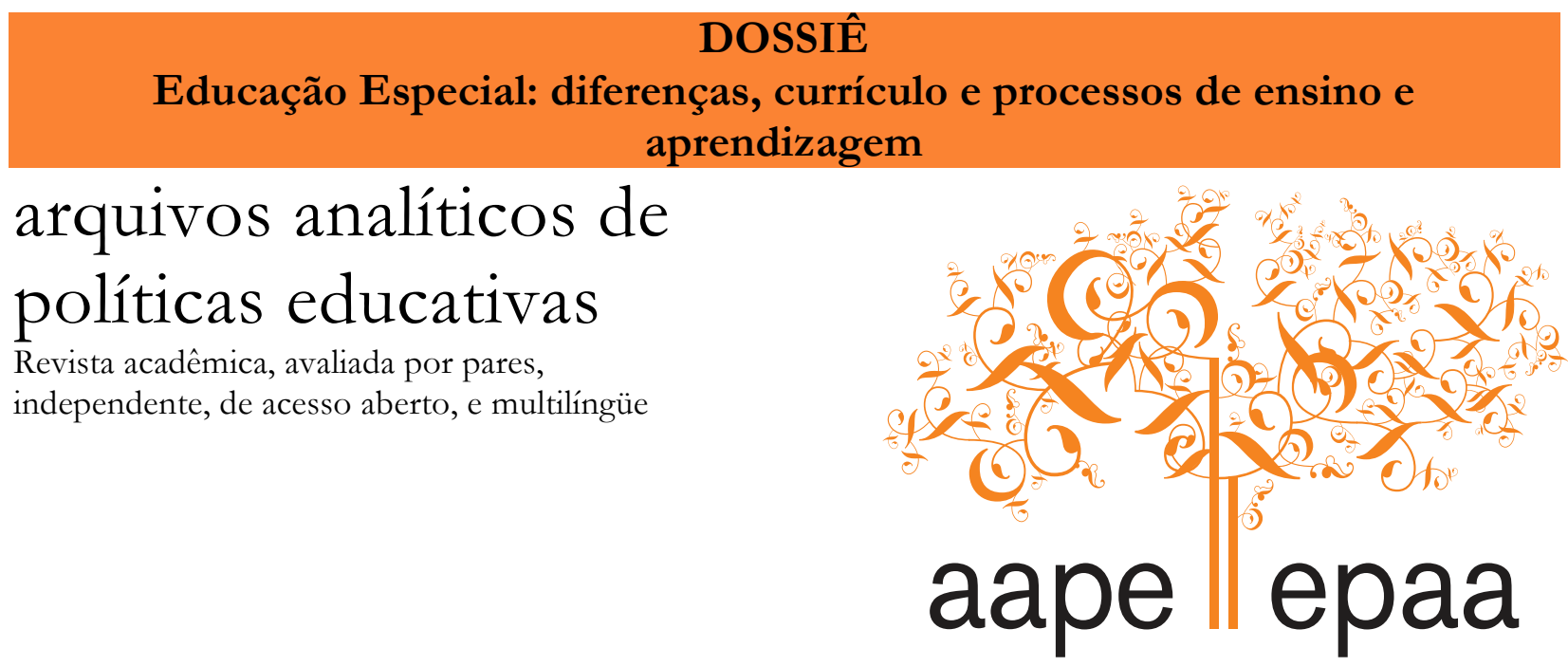

Arizona State University

ISSN 1068-2341

\title{
Influência das representações sociais do pesquisador na análise de dados de entrevistas: um estudo no campo da educação especial
}

\author{
Eduardo José Manzini \\ Universidade Estadual Paulista - Unesp de Marília \\ \& \\ Rosana Glat \\ Universidade do Estado do Rio de Janeiro - Uerj \\ Brasil
}

Citação: Manzini, E. J. \& Glat, R. (2014). Influência das representações sociais do pesquisador na análise de dados de entrevistas: um estudo no campo da educação especial. Arquivos Analíticos de Políticas Educativas, 22(79). Dossiê Educação Especial: diferenças, currículo e processos de ensino e aprendizagem. Editoras convidadas: Márcia Denise Pletsch \& Geovana Mendonça Lunardi Mendes.

http://dx.doi.org/10.14507/epaa.v22n79.2014

Resumo: Representações sociais são valores e ideias socialmente compartilhadas e transmitidas por um grupo de indivíduos. Este artigo pretende investigar como a representação pode influenciar o pesquisador na análise de uma entrevista. Os dados foram coletados com 28 estudantes que participavam de um curso sobre análise de entrevista. Estes foram divididos em três grupos, cada um recebendo o mesmo trecho de uma transcrição, porém com diferentes caracterizações sobre o entrevistado. Para o grupo 1, era um jovem que havia estudado numa escola pública; para o Grupo 2, o sujeito havia estudado numa escola especial para pessoas com deficiência intelectual; e, para o grupo 2, 
ele havia estudado numa escola particular. Aos três grupos foi solicitado que realizassem a análise e entregassem um documento escrito sobre essa análise. Os dados revelaram que os estudantes realizaram sínteses interpretativas, sínteses descritivas e análise temática. As interpretações dos dados de conteúdo indicaram que os participantes do grupo 1 e 3 atribuíram representações negativas sobre o entrevistado e/ou sobre a escola pública ou particular. Contrariamente, os participantes do Grupo 2, que receberam a descrição de que o entrevistado possuía deficiência intelectual leve, atribuíram representações positivas sobre o entrevistado e sobre a escola especializada. A conclusão indica a necessidade de formação teórica e prática para jovens pesquisadores no que se refere à análise de entrevistas para que as representações não criem resultados enviesados. A comparação dos resultados com um estudo semelhante anterior aponta uma possível transformação nas representações sobre pessoas com deficiência intelectual.

Palavras-chave: Educação Especial; Representação Social; Análise de Entrevista.

\section{Influence of researcher's social representations in analysis of interviews: a study in the field of special education}

Abstract: Social representations are values and ideas shared and transmitted by a group of individuals. This article aims to investigate how researchers' social representations can influence their analysis of an interview. Data were collected from 28 students participating in a course on interview analysis. Students were divided into three groups. Each group received the same excerpt of a transcript, but with different characterizations of the interviewee: for Group 1, it was a young man who had studied in a public school; for Group 2, the subject had attended a special education school for people with intellectual disabilities; for Group 3, he had studied at a private school. The three groups were asked to analyze the interview and submit a written document describing their analysis. The data revealed that the students carried out interpretive synthesis, descriptive synthesis, and thematic analysis. Interpretations of content data indicated that participants in Groups 1 and 3 attributed negative representations to the interviewee and/or to public or private school. In contrast, participants in Group 2, which was told that the interviewee had intellectual disabilities, attributed positive representations to the interviewee and to the special education school. The conclusion indicates the urgency for theoretical and practical training of young researchers regarding analysis of interviews so that the representations do not lead to biased results. The comparison of the results with an earlier similar study points to a probable change in representations of people with intellectual disabilities.

Keywords: Special Education; Social Representation; Analysis of Interview.

Influencia de las representaciones sociales del investigador en el análisis de los datos de las entrevistas: un estudio en el campo de la educación especial

Resumen: Las representaciones sociales son valores y ideas socialmente compartidas y transmitidas por un grupo de personas. Este artículo tiene como objetivo investigar cómo la representación puede influir el investigador en el análisis de una entrevista. Los datos fueron recolectados con 28 estudiantes que habían participado en un curso sobre análisis de la entrevista. Los estudiantes fueron divididos en tres grupos, cada uno recibiendo el mismo tramo de la transcripción, pero con diferentes caracterizaciones del entrevistado. Para el grupo 1, era un hombre joven que había estudiado en una escuela del gobierno, para el Grupo 2, el sujeto estudió en una escuela especial para personas con discapacidad intelectual, y para el grupo 3, que había estudiado en una escuela privada. Se pidió a los tres grupos para realizar el análisis de la entrevista y entregar un documento escrito sobre ella. Los datos revelaron que los estudiantes realizan síntesis interpretativas, síntesis 
descriptivas y análisis temático. Las interpretaciones de los datos de contenido indican que los participantes en el grupo 1 y 3 atribuyeron representaciones negativas para el entrevistado y / o a la escuela pública o privada. Por el contrario, los participantes en el Grupo 2, que recibieron la descripción que el entrevistado tenía una discapacidad intelectual leve, atribuyeron representaciones positivas para el entrevistado y a la escuela especializada. La conclusión indica la urgencia de la formación teórica y práctica de los jóvenes investigadores en relación con el análisis de las entrevistas a fin de que las representaciones no den lugar a resultados sesgados. La comparación de los resultados con un estudio similar anterior apunta a una posible transformación en las representaciones de las personas con discapacidad intelectual.

Palabras-clave: Educación Especial; Representación Social; Análisis de Entrevista.

\section{Introdução}

A análise de informações verbais advindas de entrevista não é uma tarefa fácil para o jovem pesquisador que está experienciando, pela primeira vez, esse procedimento. No Brasil, a entrevista é muito comum em pesquisas na área de educação. Às vezes, ela é utilizada como única forma para coletar dados, e, em outras, é um complemento para dados coletados por meio de questionários, survey, escalas (Bogdan \& Biklen, 2006), observação, análise documental (Glat \& Pletsch, 2012), entre outros.

Apesar de sua ampla utilização, a análise de entrevistas requer conhecimento teórico e treinamento prático por parte do pesquisador. Estudos têm demonstrado que a fundamentação teórica para os procedimentos de análise nem sempre está detalhada claramente nos relatórios de pesquisas de mestrado ou doutorado (Manzini, 2009; 2012). Quando especificada, o autor mais citado para respaldar a análise da entrevista tem sido Bardin (1977).

Em seu livro, Bardin (1977) apresenta vários exemplos práticos para análise de conteúdo, bem como fundamentações teóricas calcadas em autores que se serviram de entrevista para coletar dados. Entre os procedimentos apresentados pela autora, a "análise categorial" e a "análise temática" têm sido as mais privilegiadas, no Brasil, para trabalhar com as informações de entrevista. Mais recentemente, outras fundamentações teóricas têm sido propostas, começando a aparecer nos trabalhos de mestrado e doutorado em educação e educação especial tais como "discurso do sujeito coletivo" (Lefèvre, Lefèvre \& Teixeira, 2000; Lefèvre \& Lefèvre, 2003) e a "análise proposicional do discurso" (Pires, 2008). Quando as pesquisas utilizam entrevista e outros procedimentos complementares, como documentos, observação e/ou questionários, geralmente têm se encontrado, para a análise, o uso da metodologia de "triangulação de dados" (Triviños, 1987).

Independente de suas propostas específicas, todas essas abordagens teóricas têm dois denominadores comuns. Primeiro, a preocupação de que a análise de falas se transforme em um dado científico. E segundo, que os dados obtidos pela transcrição das entrevistas não sejam, simplesmente, interpretados antes que seja apresentada uma análise pormenorizada que demonstre como foi realizada tal interpretação. O objetivo é a busca de uma validade científica por meio de uma análise. E é justamente esse enfoque que diferencia uma entrevista para fins de obtenção de dados científicos de uma entrevista social ou jornalística, por exemplo.

No campo da educação especial isso não é diferente. Porém, deve-se acrescentar um novo ingrediente: o objeto de pesquisa, que pode ser pessoas com deficiência, com altas habilidades/superdotação, com autismo, ou outras necessidades especiais, as quais são, a priori, sujeitos estigmatizados. Em outras palavras, a visão que se têm desses indivíduos, já é por si só impregnada de idéias preconcebidas, geralmente, depreciativas. 
Várias produções científicas têm tentado informar e questionar os preconceitos e estigmas imputados sobre essa parcela da população, como, por exemplo, os mitos sobre os alunos com altas habilidades/superdotação (Fleith, 2009; Freitas \& Pérez, 2011), ou como ocorre a construção social da deficiência (Omote, 1990; 1994; 1999; Glat, 2009) ou, ainda, a discussão sobre sexualidade da pessoa com deficiência (Glat \& Freitas, 2007; Maia \& Ribeiro, 2010).

Representações sociais constituem um conjunto de valores, ideias, percepções, conceitos e práticas, compartilhadas e transmitidas, coletivamente, nas relações sociais de um número significativo de pessoas. Tomam forma de acordo com os diferentes contextos e momentos históricos, influenciando não só a coletividade, mas também os sujeitos em sua individualidade (Antunes, 2012; Moscovici, 2003).

A questão que se pondera é o quanto a representação, sobre um determinado indivíduo ou grupo de indivíduos - por exemplo, pessoas com deficiência -, pode influenciar o pesquisador no momento de analisar uma informação, uma fala, ou um relato proveniente desses sujeitos.

Abordagens como a etnografia tem pontuado a necessidade de o pesquisador desprover-se de seus valores para entender o objeto de estudo sob o ponto de vista da cultura do sujeito ou grupo social em investigação (Romanelli, 1998). Mas até que ponto pode-se evitar a influencia dos valores pessoais do pesquisador? Essa questão relaciona-se intimamente com a problemática do presente artigo: como os vieses e interpretações subjetivas podem emergir em uma análise de uma entrevista.

Um estudo de referência que abarcou esta problemática foi desenvolvido por Manzini (2006) com dez estudantes de cursos de mestrado e doutorado em educação. A coleta de dados foi realizada em situação de aula prática sobre análise de entrevista. Os participantes tiveram como tarefa realizar uma análise de um pequeno trecho de uma transcrição de uma entrevista sobre a inserção no mercado de trabalho de um jovem com 22 anos, para a qual poderiam utilizar os critérios que desejassem. Os dez estudantes foram divididos em três grupos e usou-se um estímulo distrativo diferente para cada grupo, alterando as características do entrevistado. A um dos grupos mencionouse tratar de um jovem que frequentava escola pública, para o outro grupo descreveu-se que o entrevistado havia estudado em uma escola privada, e para o terceiro grupo relatou-se que o entrevistado possuía deficiência intelectual leve e fora treinado em uma instituição especializada.

Sinteticamente, os resultados indicaram que alguns participantes realizaram uma síntese descritiva, outros, uma síntese descritiva e interpretativa, e, um terceiro grupo, uma análise temática dividindo e reorganizando o texto transcrito em temas e subtemas. Foi significativo o fato de que o grupo que recebeu a descrição de que o entrevistado era um jovem com deficiência intelectual se ateve a procurar em sua fala indícios ou características da deficiência, enquanto que os participantes dos outros grupos privilegiaram as informações e fatos relatados na transcrição da entrevista. $\mathrm{O}$ estudo indicou que a descrição do entrevistado como tendo deficiência intelectual influenciou a análise e interpretação dos dados.

Visando verificar se o mesmo fenômeno acontece nos dias atuais, foi realizado um novo estudo, replicando o mesmo procedimento utilizado por Manzini em 2006, o qual será descrito e discutido a seguir. Assim, o objetivo do presente estudo é investigar como jovens pesquisadores interpretam ou produzem vieses a partir de uma análise de conteúdo de dados advindos de uma entrevista transcrita.

\section{Métodos}

A amostra do estudo foi composta por 28 estudantes oriundos de cursos de graduação em pedagogia, estudantes de mestrado e doutorado em educação que participavam de um curso sobre análise de entrevista, o qual incluía uma parte prática. A coleta de dados do experimento foi o 
exercício prático que os estudantes desenvolveram nesse momento específico do curso. Como mencionado, esse designe foi o mesmo apresentado por Manzini (2006).

Os 28 estudantes foram separados em três grupos, aleatoriamente, sendo que os Grupos $1 \mathrm{e}$ 2 foram compostos por nove participantes e o Grupo 3 por dez. Para cada grupo foi entregue o mesmo trecho de transcrição utilizado por Manzini (2006) com as mesmas alterações em relação às características do entrevistado. As descrições para cada grupo $(\mathrm{G})$ foram:

G1: "Você está recebendo um pequeno trecho de transcrição de uma entrevista para análise. Trata-se de um relato de um jovem com 22 anos de idade, que estuda em uma escola governamental (ensino gratuito), tipo SENAI (indicado pela letra C., que significa centro de treinamento) para atuar em serviço de almoxarifado, office boy, dentre outras funções. Você deverá fazer a análise utilizando os critérios que desejar. Neste exercício, não existe certo ou errado. Assim sendo, você irá estabelecer o que julgar necessário para fazer a análise. Para concretizar essa análise, você deverá apresentá-la por escrito ao final do período que foi estabelecido”.

G2: "Você está recebendo um pequeno trecho de transcrição de uma entrevista para análise. Trata-se de um relato de um jovem com 22 anos de idade, de deficiência intelectual leve, que estudou em uma escola especializada (centro de treinamento, indicado pela letra C.) para atuar em serviço de almoxarifado, office boy, dentre outras funções. Você deverá fazer a análise utilizando os critérios que desejar. Neste exercício, não existe certo ou errado. Assim sendo, você irá estabelecer o que julgar necessário para fazer a análise. Para concretizar essa análise, você deverá apresentá-la por escrito ao final do período que foi estabelecido”.

G3: "Você está recebendo um pequeno trecho de transcrição de uma entrevista para análise. Trata-se de um relato de um jovem com 22 anos de idade, que estudou em uma escola particular (indicado pela letra C., que significa centro de treinamento) para atuar em serviço de almoxarifado, office boy, dentre outras funções. Você deverá fazer a análise utilizando os critérios que desejar. Neste exercício, não existe certo ou errado. Assim sendo, você irá estabelecer o que julgar necessário para fazer a análise. Para concretizar essa análise, você deverá apresentá-la por escrito ao final do período que foi estabelecido".

Os estudantes entregaram, por escrito, as suas análises ao final do exercício prático. Esse documento foi submetido à análise de conteúdo para identificar, primeiramente, se os estudantes realizaram uma síntese descritiva, interpretativa ou de outro tipo, como análise temática ou categorial, conforme os achados de Manzini (2006). Essa análise permitiu identificar e quantificar cinco categorias: 1) Síntese descritiva e interpretativa sobre o conteúdo da entrevista; 2) Síntese descritiva sobre o conteúdo da entrevista; 3) Síntese descritiva e interpretativa sobre o processo da entrevista; 4) Análise temática; 5) Análise temática e posterior síntese descritiva

O segundo tipo de análise de conteúdo teve a função de verificar se as possíveis interpretações que os estudantes realizaram relacionavam-se às alterações propositais das características do entrevistado: 1) jovem que estudou em escola pública; 2) jovem com deficiência intelectual leve que estudou em instituição especializada; 3) jovem que estudou em escola privada. Essas interpretações poderiam indicar uma representação positiva ou negativa em relação ao entrevistado e/ou em relação à sua possível adaptação ao ambiente de trabalho. 


\section{Resultados}

A primeira forma para trabalhar os documentos produzidos pelos alunos recaiu sobre o tipo de análise realizada: descritiva, interpretativa ou temática. A categoria Sintese descritiva e interpretativa sobre o conteúdo da entrevista refere-se às descrições que pontuam o conteúdo das falas e que incorporam juízo de valor às descrições. Por exemplo:

"O jovem com vinte e dois anos entrevistado apresenta em seu relato o lugar de subalternidade que ocupa na sociedade contemporânea." (Estudante 1 do Grupo 1).

"O entrevistado revela imprecisão ao descrever o horário de trabalho ...ele parece incompreender seu período de trabalho.” (Estudante 9 do Grupo 2).

"Inicialmente o entrevistado parece estar nervoso, errando o nome da sua função no trabalho.” (Estudante 19 do Grupo 3).

As frases "apresenta em seu relato um lugar de subalternidade", "ele parece incompreender" e "parece estar nervoso" atribuem um juízo de valor ou uma interpretação que enviesa a informação contida no trecho da transcrição analisada, que pode ser relacionada com a representação social ou estereótipo do sujeito (aluno de escola pública, de instituição especializada para deficiência intelectual, de escola privada).

A Sintese descritiva sobre o conteúdo da entrevista pontua o conteúdo, mas não incorpora juízo de valor à fala do entrevistado:

"o jovem exercia a função de torneiro mecânico, fabricando máquinas de vela e giz de cera... o jovem avalia que não teve proveito no treinamento realizado na instituição... o jovem considera o seu trabalho pesado, por se sujar muito e ganhar pouco.” (Estudante 26 do Grupo 3).

Como visto, neste tipo de análise, o Estudante 26 utiliza na sua descrição palavras que constavam na transcrição da entrevista: "trabalho pesado", "suja-se muito", "ganha-se pouco".

$\mathrm{Na}$ Sintese descritiva e interpretativa sobre o processo da entrevista o enfoque recai sobre a condução da entrevista pelo entrevistador. As interpretações encerram possíveis imperfeições nas perguntas ou nas respostas. Para ilustrar essa categoria seguem alguns exemplos:

“O pesquisador usa jargão para se comunicar... algumas perguntas não são objetivas..." (Estudante 8 do Grupo 1).

"Existem perguntas informais feitas pelo entrevistado... ele (o entrevistador) não é claro nas perguntas..." (Estudante 15 do Grupo 2).

A categoria Análise temática consiste em apresentar uma classificação do conteúdo em temas. Para cada tema, os estudantes ilustraram a classificação com as falas selecionadas da transcrição. Segue um trecho da análise temática:

"Profissão: torneiro mecânico

Tarefas: realizar furagem, marcagem de chapa... 
Jornada de trabalho: das 6 às 7 horas...

Opinião sobre o trabalho: está muito pesado!

Relação com os demais funcionários: [...].” (Estudante 28 do Grupo 3).

A categoria Análise temática e posterior sintese descritiva consiste em uma análise temática, previamente descrita, adicionada de um texto descritivo construído pelos alunos (síntese descritiva). Portanto, trata-se da construção de um sistema de categorias para respaldar uma síntese, considerado o tipo de proposta que mais se reveste de validade científica. A partir da síntese descritiva seria possível entrar no nível de discussão e interpretação dos dados, tarefa que não foi solicitada no exercício proposto aos alunos participantes da pesquisa. Tabela 1.

As cinco categorias identificadas foram quantificadas e o resultado pode ser observado na

Tabela 1

Quantificação das categorias identificadas nos documentos apresentados pelos estudantes.

\begin{tabular}{lcccc}
\hline Categorias & G1 & G2 & G3 & Total \\
\hline Síntese descritiva e interpretativa sobre o conteúdo da entrevista & 4 & 6 & 3 & 13 \\
Síntese descritiva e interpretativa sobre o processo da entrevista & 5 & 2 & 4 & 11 \\
Análise temática e posterior síntese descritiva & & 1 & 1 & 2 \\
Síntese descritiva sobre o conteúdo da entrevista & & & 1 & 1 \\
Análise temática & & & 1 & 1 \\
\hline Total & $\mathbf{9}$ & $\mathbf{9}$ & $\mathbf{1 0}$ & $\mathbf{2 8}$ \\
\hline
\end{tabular}

Fonte: própria dos autores

Como mostrado na Tabela 1, as sínteses descritivas e interpretativas (seja sobre o conteúdo ou o processo da entrevista) foram as opções mais comuns nas atividades de análise de uma transcrição de uma entrevista feitas pelos participantes. As demais categorias foram muito pouco utilizadas pelos estudantes.

A segunda de maior interesse era verificar se as interpretações que os estudantes realizaram em função das alterações propositais das características do entrevistado indicariam uma representação positiva ou negativa em relação ao sujeito e/ou ao ambiente de trabalho.

Esse tipo de interpretação é visível nos casos em que os estudantes se referiram ao conteúdo, ou seja, na categoria Síntese descritiva e interpretativa sobre o conteúdo da entrevista. Treze participantes compuseram suas análises dentro dessa categoria, que corresponde à primeira linha da Tabela 1 .

Quatro estudantes do Grupo 1, que receberam a informação de que o entrevistado havia estudado em uma escola pública, descreveram e interpretaram o conteúdo da entrevista. Alguns trechos dessas interpretações foram selecionados:

"Podemos notar que existe um erro na escolha do curso, pois o aluno não gosta do trabalho de Office Boy e não pode trabalhar em almoxarifado porque é alérgico a pó." (Estudante 2).

“O entrevistado não tinha domínio prévio sobre a profissão que desempenha atualmente.” (Estudante 3).

“Ele não pensa em exercer as funções nas quais se formou." (Estudante 3).

"Tendo como critério a socialização, o ambiente laboral permite concluir que o jovem possui pouca interação com colegas de trabalho.” (Estudante 4). 
“Não há convivência fora do ambiente de trabalho.”(Estudante 4).

"Ocorreu falta de compatibilidade - entre o treinamento e o trabalho realizado devido alegar problemas de saúde, como alergia.” (Estudante 4).

Seis estudantes do Grupo 2, em que entrevistado era descrito como tendo deficiência intelectual, realizaram descrições e interpretações sobre o conteúdo da entrevista, conforme os exemplos a seguir:

"(o entrevistado) revela ter trabalhado anteriormente em uma rede de supermercados como auxiliar de escritório por certo período curto de 3 meses. Isso parece revelar que ele foi incluído no mercado de trabalho conforme orientação fornecida pela escola especializada onde estudou." (Estudante 10).

"Ele sabe contar passo a passo (as atividades que realiza) no seu trabalho como torneiro mecânico, identifica o número de pessoas com quem trabalha e a pessoa que ensinou o ofício atual." (Estudante 10).

"Percebemos nesse relato que ele interage muito bem como dono da empresa." (Estudante 10).

"O sujeito apresenta experiência. O fato de já ter experiência contribuiu para sua adaptação no mercado de trabalho.” (Estudante 12).

"A instituição (especializada) não analisa os interesses e desejos do sujeito na oferta do curso, pois o sujeito alega que aprendeu serviço de estoquista, mas não pode trabalhar nisso porque é alérgico a pó" (Estudante 12).

"O sujeito apresenta insatisfação com as condições de trabalho, pois se suja e ganha pouco - noção de dinheiro.” (Estudante 12).

“Nota-se também não haver satisfação salarial (ganha pouco).” (Estudante 18).

"Esse jovem passou por um Centro de Treinamento que contribuiu para sua autonomia." (Estudante 17).

"Percebe-se que as empresas em que trabalhou tinham algum processo de inclusão para pessoas que apresentam necessidades especiais.” (Estudante 17).

"Podemos dizer que este jovem está em busca de sua emancipação e preparando-se para os obstáculos...” (Estudante 17).

"O entrevistador retoma a conversa sobre ser torneiro mecânico, então, percebe-se a boa relação do entrevistado com as máquinas, com a produção de giz de cera e velas, e com as relações pessoais com o patrão e demais colegas." (Estudante 18).

“O entrevistado revela imprecisão (ao definir) o horário de trabalho.... ele parecer incompreender seu período de trabalho." (Estudante 10). 
"A fala sobre o horário de trabalho é confusa." (Estudante 11).

Os estudantes do Grupo 3 receberam a informação de que o entrevistado havia estudado em uma escola particular. Desse grupo, três participantes realizaram a interpretação de conteúdo.

"Inicialmente o entrevistado parece estar nervoso, errando o nome de sua última função... mostrou também que possui baixa autoestima, quando relatou que não permaneceu no trabalho anterior porque não estava se adaptando...” (Estudante 19).

"Embora seja um ambiente (de trabalho) amistoso, as relações são apenas profissionais." (estudante 20).

"Pareceu que o estudante estava nervoso ao responder." (estudante 21).

\section{Discussão}

A primeira questão para discussão se refere ao tipo de análise adotada pelos participantes do estudo para interpretar a transcrição de uma entrevista. O processo de análise consiste em separar as partes de um todo, reorganizando o material, neste caso verbal, em temas, categorias ou de alguma outra forma para que o objetivo de pesquisa seja atingido. Com a elaboração de um sistema de análise é possível apresentar o material (dados brutos) para outros pesquisadores mais experientes (André, 1983) ou para “juízes” que irão referendar cientificamente o sistema de classificação. Posteriormente, pode-se, então, proceder a uma síntese e interpretação dos dados.

Entretanto, verificou-se que 25 participantes (89\%) produziram apenas uma síntese, sem criar um sistema de classificação para realizar a análise, ou seja, separação e organização das falas para entender e interpretar o conteúdo. Aliás, a própria palavra análise é frequentemente confundida pelos estudantes, que a entendem como sinônimo de interpretação de dados. O perigo de o pesquisador pular a fase de análise e constituir, de imediato, uma síntese é separar somente aquilo que mais chama a atenção, que é mais polêmico, deixando de lado informações que não se sobressaem, mas cujas mensagens latentes podem estar presentes, porém, encobertas a uma primeira leitura. Além do mais, é sabido que, à primeira vista, os dados que o pesquisador prontamente destaca dependerá do seu olhar, de suas concepções e representações sobre o objeto ou fenômeno de estudo.

Talvez um dos dados mais importantes deste experimento seja que somente três participantes $(10 \%)$ apresentaram uma análise temática. Certamente, o fato de os estudantes dirigirem-se diretamente para a síntese demonstra que o grupo não dominava procedimentos e técnicas de análise de entrevistas e, exatamente por isso, haviam procurado o referido curso. Porém, cerca de 60\% do grupo era constituído por mestrandos e doutorandos, e mesmo assim mostraramse pouco preparados para lidar com dados advindos de entrevistas.

Esses resultados corroboram o estudo original de Manzini (2006). Mesmo tendo nesse primeiro estudo uma amostra mais reduzida, os participantes elaboraram as mesmas categorias aqui discutidas: síntese descritiva, interpretativa ou temática. Não há dúvida que o pesquisador, que pretende trabalhar com entrevista, necessitará de formação teórica e prática para realizar o tratamento dos dados coletados. Infelizmente, essa demanda nem sempre é devidamente valorizada nos nossos programas de pós-graduação em educação. Paradoxalmente, conforme apontado no 
início deste texto, a entrevista é um dos procedimentos de coleta de dados mais comuns nas pesquisas de mestrado e doutorado da área.

Uma segunda questão que vale ser pontuada é por que 11 participantes (39\%) realizaram uma análise sobre o processo de entrevista e não sobre o conteúdo? Com certeza, esse dado está intimamente relacionado à dinâmica do curso, no âmbito do qual esse estudo foi realizado. Em outras palavras, os participantes estavam sob o controle da programação e estruturação do curso. $\mathrm{O}$ conteúdo inicial se voltava para definir e conceituar entrevista e, posteriormente, como elaborar um roteiro e utilizá-lo em uma entrevista. Portanto, não havia ainda sido ministrado o conteúdo programático relacionado à como realizar "análise de conteúdo". Dessa forma, os estudantes utilizaram os conhecimentos já adquiridos no curso para realizar análise de dados solicitada. Essa tendência não foi encontrada em Manzini (2006), pois, a coleta dos documentos em resposta à tarefa solicitada ocorreu logo no início de um curso de curta duração sobre análise de entrevista. Novamente, constata-se a influência das ideias ou conhecimentos preconcebidos, ou seja, os participantes utilizaram as informações já obtidas no curso para realizar a análise.

A terceira questão de discussão se refere ao objetivo maior do estudo: que vieses e interpretações equivocadas podem emergir em uma análise de uma entrevista cujo pesquisador traz uma representação social, positiva ou negativa, sobre o sujeito de investigação?

Observando os trechos selecionados de cada um dos grupos, pode-se verificar que o Grupo 1 - que recebeu a informação de que o jovem entrevistado havia estudado em uma escola pública imputou ao entrevistado o maior número de representações negativas: erro na escolha do curso, falta de domínio prévio sobre a profissão, não pensava em exercer as funções para qual fora treinado, possuía pouca interação com colegas de trabalho, etc..

As sínteses interpretativas apresentadas pelo Grupo 3 - que recebeu a informação de que o jovem entrevistado havia estudado em uma escola particular - também foram repletas de representações negativas em relação ao sujeito e ao ambiente: o entrevistado parecia estar nervoso; demonstrou baixa autoestima; embora o ambiente de trabalho fosse amistoso, as relações pessoais eram apenas profissionais.

Os dados apresentados pelo Grupo 2, que recebeu a informação de que o jovem possuía deficiência intelectual leve, entretanto, foram totalmente diferentes. As análises dos participantes da pesquisa revelaram, quase totalmente, representações positivas em relação ao entrevistado. As duas análises que revelaram representações negativas se referiram à descrição do horário de trabalho: o entrevistado parecer não compreender seu período de trabalho; a "fala" sobre o horário de trabalho está confusa.

É interessante observar que as representações positivas feitas por esse grupo também foram extensivas à instituição especializada que havia lhe proporcionado o treinamento: ele foi incluído no mercado de trabalho conforme orientação fornecida pela escola especializada onde estudou; esse jovem passou por um Centro de Treinamento que contribuiu para sua autonomia. Porém, em alguns casos, a instituição recebeu críticas: "A instituição (especializada) não analisa os interesses e desejos do sujeito na oferta do curso, pois o sujeito alega que aprendeu serviço de estoquista, mas não pode trabalhar nisso porque é alérgico a pó” (Estudante 12). Percebe-se aqui que a falha é atribuída à instituição e não ao entrevistado.

Os participantes também teceram comentários positivos em relação às empresas: elas tinham algum processo de inclusão para pessoas que apresentam necessidades especiais. Entretanto, a maioria das atribuições positivas se direcionou para o pretenso jovem com deficiência intelectual leve: 1) ele sabe contar passo a passo (as atividades que realiza) no seu trabalho; 2) ele identifica o número de pessoas com quem trabalha; 3) ele interage muito bem com o dono da empresa; 4) ele apresenta experiência num trabalho anterior, o que contribuiu para sua adaptação no mercado de trabalho; 5) ele apresenta insatisfação com as condições de trabalho, pois se suja e ganha pouco, o 
que demonstra ter noção sobre dinheiro; 6) ele está em busca de sua emancipação e preparando-se para os obstáculos; 7) ele tem uma boa relação com as máquinas, com a produção de giz de cera e velas, e com as relações pessoais com o patrão e demais colegas.

Sobre esse ponto, é interessante constatar que os dados coletados no presente estudo indicam uma tendência oposta à apontada por Manzini (2006). Naquele estudo, as atribuições em relação ao entrevistado com deficiência intelectual leve foram todas negativas, apesar de as condições de coleta de dados fossem bastante semelhantes e o material para análise fosse o mesmo.

Embora não tenha havido um controle sistemático de variáveis nas duas amostras, que permita uma comparação cientificamente constatada (até porque não havia sido essa a intenção inicial), os resultados apontam uma possível mudança de concepção de uma representação negativa para uma representação mais positiva em relação à inclusão da pessoa com deficiência intelectual no mercado de trabalho. Salienta-se que os dados de Manzini (2006) foram coletados há mais de sete anos. E, os últimos anos, o Brasil foi marcado por uma série de transformações legais e sociais que certamente influenciaram a representação e expectativas em relação a esses indivíduos.

É inegável hoje, a consolidação da política de Educação Inclusiva pelo Governo Federal. Uma série de dispositivos legais (Brasil, 2007; 2008; 2009; 2009a; 2011, entre outros) e fontes de financiamento direcionadas vêm gradativamente transformando a estrutura dos programas de atendimento a alunos com deficiências e outras necessidades educacionais especiais, bem como a organização dos sistemas escolares, de modo geral. A própria área de Educação Especial, da qual a grande maioria dos participantes em ambos os estudos é originária, vem sofrendo um intenso processo de ressignificação de sua proposta e objetivos programáticos (Glat \& Blanco, 2011; Glat \& Pletsch, 2012).

Além do campo educacional, faz parte deste cenário uma ampla articulação de políticas, programas e ações visando à inclusão social de pessoas com deficiências, com destaque para o Plano Nacional dos Direitos da Pessoa com Deficiência, também conhecido como Plano Viver sem Limites (Brasil, 2011a). A sua promulgação deu maior força à implementação de legislação anterior, inclusive no campo da inclusão no mercado de trabalho (tema da entrevista proposta) como a Lei Federal 8.213/91 de 24/07/91 (Brasil, 1991), conhecida como a "Lei de Cotas".

Independente das políticas públicas e garantias legais é preciso, também, reconhecer o grande impacto causado pelas as campanhas publicitárias, desenvolvidas pelos Ministérios da Justiça, Educação, Trabalho e Esporte, voltadas para a divulgação e esclarecimento dos direitos das pessoas com deficiência. Tais campanhas, acampadas também pela iniciativa privada têm colocado as pessoas com deficiência constantemente na mídia, na TV, blogs, redes sociais, dentre outros meios de comunicação, e vem, provocando, gradativamente, uma transformação nas representações e opinião da população.

Provavelmente, estes fatores contribuíram para que o discurso em relação a pessoas com deficiência tenha se alterado nos documentos apresentados pelos participantes da pesquisa, se comparados com o estudo anterior. Curiosamente, a representação veiculada sobre o sujeito com deficiência intelectual, ao contrário do senso comum, foi bastante generosa e produziu o que tem sido chamado de um discurso "politicamente correto". Assim, enquanto que os participantes dos grupos 1 e 3 produziram, em sua maioria, um discurso permeado de representações negativas em relação ao treinamento e as dificuldades do jovem de 22 anos em busca de emprego, os do Grupo 3 atenuaram as críticas, tentaram buscar interpretações que não desmerecessem o trecho da entrevista - objeto de análise - e construíram um texto impregnado de representações positivas em relação tanto ao entrevistado quanto a instituição educacional especializada.

${ }^{1}$ Esta prevê a contratação de dois a $5 \%$ de pessoas com deficiência em empresas com mais de 100 


\section{Considerações finais}

Em síntese, os dados coletados revelaram, claramente, que representações sociais dos pesquisadores sobre os sujeitos da pesquisa podem alterar como esses são percebidos, o que repercute na análise de suas falas em entrevistas. Isso ficou comprovado no estudo de Manzini (2006) - que mostrou tendências para interpretações negativas sobre o entrevistado descrito com tendo deficiência intelectual - e na presente pesquisa - cujos resultados indicaram que a maioria das interpretações otimizou as qualidades do mesmo jovem.

Uma segunda conclusão, inferida dos resultados obtidos, é que as concepções sobre a inclusão da pessoa com deficiência na sociedade podem estar passando um momento de alteração na realidade brasileira, pelo menos entre os estudantes e profissionais de educação. Percebe-se nas análises dos participantes, conforme mencionado anteriormente, uma predominância de interpretações positivas a respeito de inclusão no trabalho de um jovem com deficiência intelectual em comparação com o estudo original realizado há quase uma década atrás. Neste sentido, seria interessante a replicação desses procedimentos em futuras pesquisas, com maior controle experimental sobre a composição da amostra, incluindo, por exemplo, estudantes que não são da área de Educação Especial.

Porém, a contribuição maior que esse estudo traz é a comprovação da necessidade de formação metodológica-conceitual de pesquisadores para enfrentar o desafio de utilizar procedimentos sistemáticos de análise, cuidando para que os vieses possam também ser evitados para que os dados oriundos de entrevista possam ser trabalhados e validados cientificamente.

\section{Referências}

André, M. E. D. A. (1983). Texto, contexto e significados: algumas questões na análise de dados qualitativos. Cadernos de Pesquisa, 45, 66-71.

Antunes, K. C. V. (2012). História de Vida de alunos com deficiência intelectual: percurso escolar e a constituição do sujeito. Tese de Doutorado em Educação, Universidade do Estado do Rio de Janeiro.

Bardin, L. (1977). Análise de conteúdo. Lisboa: Edições 70.

Bogdan, R. C. \& Biklen, S. K. (2006). Investigação qualitativa em educação: uma introdução à teoria e aos métodos. Porto: Porto Editora. (Coleção Ciências da Educação).

Brasil. (1991). Presidência da República. Casa Civil. Dispõe sobre os Planos de Benefícios da Previdência Social e dá outras providências. http://www.planalto.gov.br/ccivil_03/leis/18213cons.htm

Brasil. (2007) Plano de Desenvolvimento da Educação (PDE). Brasília, DF. www.fundabrinq.org.br

Brasil. (2008). Ministério da Educação. Secretaria de Educação Especial. Política Nacional de Educação Especial na Perspectiva da Educaşão Inclusiva. Brasília: MEC. http://portal.mec.gov.br/arquivos/pdf/politicaeducespecial.pdf

Brasil. (2009). Decreto $N^{0}$ 6.949, de 25 de agosto de 2009. Promulga a Convenção Internacional sobre os Direitos das Pessoas com Deficiência e seu Protocolo Facultativo, assinados em Nova York, em 30 de março de 2007. Brasília, DF.

Brasil. (2009a) Resolução CNE / CEB n 4 de outubro de 2009 - Institui Diretrizes Operacionais para o

Atendimento Educacional Especializado na Educação Básica, modalidade Educação

Especial. portal.mec.gov.br/seesp 
Brasil. (2011). Decreto 7611 de 17 de novembro de 2011 - Dispõe sobre a Educação Especial, o atendimento educacional especializado e dá outras providências. http://www.planalto.gov.br/ccivil_03/_Ato2011-2014/2011/Decreto/D7611.htm

Brasil. (2011a). Decreto 7612 de 17 de novembro de 2011 - Institui o Plano Nacional dos Direitos da Pessoa com Deficiência - Plano Viver sem Limite. http://www.planalto.gov.br/ccivil_03/_Ato2011-2014/2011/Decreto/D7612.htm

Fleith, D. S.(2009). Mitos e fatos sobre os superdotados. In: O. FÁVERO et al. (Org.). Tornar a educação inclusiva (pp.199-212). Brasília: UNESCO \&AnPEd.

Freitas, S. N. \& Pérez, S. G. P. B. (2011). Altas habilidades/superdotação: respostas a 30 perguntas. Porto Alegre: Redes Editora.

Glat, R. \& Freitas, R. C. (2007). Sexualidade e deficiência mental: pesquisando, refletindo e debatendo sobre o tema. $3^{\text {a }}$ edição. Rio de Janeiro: 7 Letras.

Glat, R. (2009). Somos iguais a vocês - depoimentos de mulheres com deficiência mental. $2^{\mathrm{a}}$ edição. Rio de Janeiro: Sete Letras

Glat, R. \& Blanco, L. de M. V. (2011).Educação Especial no contexto de uma Educação Inclusiva. In: R. Glat, (Org.), Educação Inclusiva: cultura e cotidiano escolar. (pp. 15-35). $2^{\mathrm{a}}$ edição, Rio de Janeiro: Sete Letras,

Glat, R. \& Pletsch, M. D. (2012) Inclusão escolar de alunos com necessidades especiais.2a edição. Rio de Janeiro: EdUERJ.

Lefèvre, F; Lefèvre, A. M. C. \& Teixeira, J. J. V. (2000). O discurso do sujeito coletivo: uma nova abordagem metodológica em pesquisa qualitativa. Caxias do Sul: Educs.

Lefevre, F.\& Lefevre, A. M. C. (2003). O discurso do sujeito coletivo: um novo enfoque em pesquisa qualitativa - desdobramentos. Caxias do Sul: Educs.

Maia, A. C. B. \& Ribeiro, P. R. M. (2010). Desfazendo mitos para minimizar o preconceito sobre a sexualidade de pessoas com deficiência. Revista Brasileira de Educação Especial, 16(2), 159-176.

Manzini, E. J. (2006). Considerações sobre a entrevista para a pesquisa social em educação especial: um estudo sobre análise de dados. In: D. M. Jesus, C. R. Baptista \& S. L. Victor. Pesquisa e educação especial: mapeando produções (pp.361-386).Vitória: UFES.

Manzini, E. J. (2009). Análise do uso da entrevista em dissertações e teses em educação especial. In: C. R. Baptista \& D. M. Jesus (Org.), Conhecimento e margens: ação pedagógica e pesquisa em educação especial (pp.167-188). Porto Alegre: Mediação/CDV/FACITEC.

Manzini, E. J. (2012). Uso da entrevista em dissertações e teses produzidas em um programa de pós-graduação em educação. Revista Percurso (Online), 4, 149-171.

Moscovici (2003). Representações sociais: investigações em psicologia social. Petrópolis-RJ: Vozes.

Omote, S.(1990). Aparência e competência em educação especial. In: Temas em educação especial (pp.11-26). São Carlos: UFSCar.

Omote, S. (1994). Deficiência e não-deficiência: recortes do mesmo tecido. Revista Brasileira de Educação Especial, 1(2), 65-73.

Omote, S. (1999). Deficiência: da diferença ao desvio. In: E. J. Manzini \& P. R. Brancatti (Org.), Educação especial e estigma (pp.3-21). Marília: NAC Publicações.

Pires, J. (2008). Teoria e prática da análise proposicional do discurso. João Pessoa: Idéia.

Romanelli, G. (1998). A entrevista antropológica: troca e alteridade. In: G. Romanelli \& Z. M. M Bisoli-Alves. Diálogos metodológicos sobre prática de pesquisa. (pp.119-133. Ribeirão Preto: Legis Summa.

Triviños, A. N. S. (1987). Introdução à pesquisa em ciências sociais: a pesquisa qualitativa em educação. São Paulo: Atlas. 


\title{
Eduardo José Manzini
}

\section{Sobre os Autores}

Professor adjunto da Universidade Estadual Paulista "Júlio de Mesquista Filho", Faculdade de Filosofia e Ciências, Unesp, Marília. Docente do Programa de Pós-Graduação em Educação na Unesp de Marília, possui graduação em Psicologia pela Unimep (1983), mestrado em Educação Especial pela UFSCar (1989); doutorado em Psicologia pela USP-SP (1985), livre-docência pela Unesp de Marília (2008), pós-doutorado pela UERJ (2014). manzini@marilia.unesp.br

\section{Rosana Glat}

Professora Associada da Faculdade de Educação e do Programa de Pós-Graduação em Educação da Universidade do Estado do Rio de Janeiro (PROPEd/UERJ), Diretora da Faculdade de Educação e Vice-presidente da ABPEE e pesquisadora CNPq e Faperj. rglat@terra.com.br

\section{Sobre as Editoras Convidadas}

\begin{abstract}
Márcia Denise Pletsch
Professora do Programa de Pós-Graduação em Educação, Contextos Contemporâneos e Demandas Populares (PPGEduc) na linha de pesquisa Estudos Contemporâneos e Práticas Educativas e do Departamento Educação e Sociedade da Universidade Federal Rural do Rio de janeiro. marciadenisepletsch@gmail.com Informação biográfica: É pesquisadora na área de Educação Especial, atuando na formação de professores e de novos pesquisadores. É líder do Grupo de Pesquisa (CNPq) Observatório de Educação Especial e inclusão escolar: práticas curriculares e processos de ensino e aprendizagem e, por meio de convênio interinstitucional entre a UFRRJ e a Universidade do Estado do Rio de Janeiro (UERJ), também é líder do grupo de pesquisa Inclusão e aprendizagem de alunos com necessidades educacionais especiais: práticas pedagógicas, cultura escolar e aspectos psicossociais. Atualmente, coordena o Programa Observatório da Educação da CAPES com projeto de pesquisa em rede na área de deficiência intelectual envolvendo a Universidade Estadual de Santa Catarina (UDESC), Universidade do Vale do Itajaí (UNIVALI); e coordena também pesquisas financiadas pela FAPERJ na área de deficiência múltipla. É autora do livro "Repensando a inclusão escolar: diretrizes políticas, práticas curriculares e deficiência intelectual” e, em colaboração com Rosana Glat, do livro "Inclusão escolar de alunos com necessidades especiais". Organizou em parceria com outros pesquisadores, entre outros, os livros "Estratégias educacionais diferenciadas para alunos com necessidades especiais" e "Educação Especial e inclusão escolar: reflexões sobre o fazer pedagógico". Tem mais de vinte artigos publicados em revistas científicas nacionais e internacionais.
\end{abstract}

Geovana Mendonça Lunardi Mendes - Professora do Programa de Pós-Graduação em Educação e do Departamento de Pedagogia da Universidade do Estado de Santa Catarina (PGEUDESC). geolunardi@gmail.com É pesquisadora na área de Educação Especial e dos Estudos Curriculares. Realizou Pós-Doutorado na Argentina e nos Estados Unidos da América, na área de Currículo e Novas Tecnologias, na Universidad de San Andres em Buenos Aires e em Ashland University, em Ohio. É pesquisadora coordenadora de diferentes projetos de investigação e participa como pesquisadora convidada em projetos de pesquisa nacionais e internacionais. Suas pesquisas e produções têm sido voltadas para 
área de Currículo e práticas escolares, em especial, as questões relativas as mudanças, novas tecnologias e inovações curriculares no espaço escolar, e também as práticas curriculares voltadas a inclusão de sujeitos com deficiência. Atualmente é a Coordenadora Nacional do Consórcio "Educação e Diversidade" do programa CAPES. FIPSE de Cooperação Internacional, envolvendo a Universidade Federal Rural do Rio de Janeiro, no Brasil e Georgetown College, Ashland University e Brighman Young University nos Estados Unidos e também do Projeto de Pesquisa: Aulas conectadas: mudanças curriculares e aprendizagem colaborativa nas escolas do PROUCA em Santa Catarina, com financiamento do CNPq e do Projeto Observatório de Práticas Escolares com financiamento da FAPESC. É coordenadora do Programa de Pós-graduação em Educação, Mestrado e Doutorado, da FAED, UDESC. Coordena o Observatório da Educação: Tablets, Computadores e Laptops, aprovado no Edital OBEDUC/CAPES. Entre suas atuais produções podem ser destacadas o livro intitulado "Objetos Pedagógicos: uma experiência inclusive em oficinas de Artes", em parceria com mais duas autoras e a organização do Livro "Deficiência e Escolarização: novas perspectivas de analise", hoje na segunda edição. É autora de inúmeros capítulos e artigos publicados em periódicos.

\section{DOSSIE}

\section{Educação Especial: diferenças, currículo e processos de ensino e} aprendizagem

arquivos analíticos de políticas educativas

Volume 22 Número $79 \quad 11$ de agosto de 2014

ISSN 1068-2341

\section{(c) \\ SORERIGHIS RESEREED O Copyright e retido pelo/a o autor/a (ou primeiro co-autor) que outorga o} direito da primeira publicação à revista Arquivos Analíticos de Políticas Educativas. Más informação da licença de Creative Commons encontram-se em http://creativecommons.org/licenses/by-nc-nd/2.5. Qualquer outro uso deve ser aprovado em conjunto pelo/s autor/es e por AAPE/EPAA. AAPE/EPAA é publicada por Mary Lou Fulton Institute Teachers College da Arizona State University. Os textos publicados em AAPE são indexados por CIRC (Clasificación Integrada de Revistas Científicas, Espanha) DIALNET (Espanha),Directory of Open Access Journals, Education Full Text (H.W. Wilson), EBSCO Education Research Complete, , ERIC, , QUALIS A2 (Brasil), SCImago Journal Rank; SCOPUS, SOCOLAR (China). Contribua com comentários e sugestões a http://epaa.info/wordpress/ ou para Gustavo E. Fischman fischman@,asu.edu.

Curta a nossa comunidade EPAA's Facebook https://www.facebook.com/EPAAAAPE e Twitter feed@epaa_aape. 


\section{arquivos analíticos de políticas educativas conselho editorial}

Editor: Gustavo E. Fischman (Arizona State University)

Editores Associados: Rosa Maria Bueno Fisher e Luis A. Gandin

(Universidade Federal do Rio Grande do Sul)

Dalila Andrade de Oliveira Universidade Federal de Minas Gerais, Brasil

Paulo Carrano Universidade Federal Fluminense, Brasil

Alicia Maria Catalano de Bonamino Pontificia Universidade Católica-Rio, Brasil

Fabiana de Amorim Marcello Universidade Luterana do Brasil, Canoas, Brasil

Alexandre Fernandez Vaz Universidade Federal de Santa Catarina, Brasil

Gaudêncio Frigotto Universidade do Estado do Rio de Janeiro, Brasil

Alfredo M Gomes Universidade Federal de Pernambuco, Brasil

Petronilha Beatriz Gonçalves e Silva Universidade Federal de São Carlos, Brasil

Nadja Herman Pontificia Universidade Católica -Rio Grande do Sul, Brasil

José Machado Pais Instituto de Ciências Sociais da Universidade de Lisboa, Portugal

Wenceslao Machado de Oliveira Jr. Universidade Estadual de Campinas, Brasil
Jefferson Mainardes Universidade Estadual de Ponta Grossa, Brasil

Luciano Mendes de Faria Filho Universidade Federal de Minas Gerais, Brasil

Lia Raquel Moreira Oliveira Universidade do Minho, Portugal

Belmira Oliveira Bueno Universidade de São Paulo, Brasil

António Teodoro Universidade Lusófona, Portugal

Pia L. Wong California State University Sacramento, U.S.A

Sandra Regina Sales Universidade Federal Rural do Rio de Janeiro, Brasil

Elba Siqueira Sá Barreto Fundação Carlos Chagas, Brasil

Manuela Terrasêca Universidade do Porto, Portugal

Robert Verhine Universidade Federal da Bahia, Brasil

Antônio A. S. Zuin Universidade Federal de São Carlos, Brasil 


\section{education policy analysis archives editorial board}

Editor Gustavo E. Fischman (Arizona State University)

Associate Editors: Audrey Amrein-Beardsley (Arizona State University), Rick Mintrop, (University of California, Jeanne M. Powers (Arizona State University)

Jessica Allen University of Colorado, Boulder

Gary Anderson New York University

Michael W. Apple University of Wisconsin, Madison

Angela Arzubiaga Arizona State University

David C. Berliner Arizona State University

Robert Bickel Marshall University

Henry Braun Boston College

Eric Camburn University of Wisconsin, Madison

Wendy C. Chi* University of Colorado, Boulder

Casey Cobb University of Connecticut

Arnold Danzig Arizona State University

Antonia Darder University of Illinois, UrbanaChampaign

Linda Darling-Hammond Stanford University

Chad d'Entremont Strategies for Children

John Diamond Harvard University

Tara Donahue Learning Point Associates

Sherman Dorn University of South Florida

Christopher Joseph Frey Bowling Green State University

Melissa Lynn Freeman* Adams State College

Amy Garrett Dikkers University of Minnesota

Gene V Glass Arizona State University

Ronald Glass University of California, Santa Cruz

Harvey Goldstein Bristol University

Jacob P. K. Gross Indiana University

Eric M. Haas WestEd

Kimberly Joy Howard* University of Southern California

Aimee Howley Ohio University

Craig Howley Ohio University

Steve Klees University of Maryland

Jaekyung Lee SUNY Buffalo
Christopher Lubienski University of Illinois, UrbanaChampaign

Sarah Lubienski University of Illinois, UrbanaChampaign

Samuel R. Lucas University of California, Berkeley

Maria Martinez-Coslo University of Texas, Arlington

William Mathis University of Colorado, Boulder

Tristan McCowan Institute of Education, London

Heinrich Mintrop University of California, Berkeley

Michele S. Moses University of Colorado, Boulder

Julianne Moss University of Melbourne

Sharon Nichols University of Texas, San Antonio

Noga O'Connor University of Iowa

João Paraskveva University of Massachusetts, Dartmouth

Laurence Parker University of Illinois, UrbanaChampaign

Susan L. Robertson Bristol University

John Rogers University of California, Los Angeles

A. G. Rud Purdue University

Felicia C. Sanders The Pennsylvania State University

Janelle Scott University of California, Berkeley

Kimberly Scott Arizona State University

Dorothy Shipps Baruch College/CUNY

Maria Teresa Tatto Michigan State University

Larisa Warhol University of Connecticut

Cally Waite Social Science Research Council

John Weathers University of Colorado, Colorado Springs

Kevin Welner University of Colorado, Boulder

Ed Wiley University of Colorado, Boulder

Terrence G. Wiley Arizona State University

John Willinsky Stanford University

Kyo Yamashiro University of California, Los Angeles

* Members of the New Scholars Board 


\section{archivos analíticos de políticas educativas consejo editorial}

Editor: Gustavo E. Fischman (Arizona State University)

Editores. Asociados Alejandro Canales (UNAM) y Jesús Romero Morante (Universidad de Cantabria)

Armando Alcántara Santuario Instituto de Investigaciones sobre la Universidad y la Educación, UNAM México

Claudio Almonacid Universidad Metropolitana de Ciencias de la Educación, Chile

Pilar Arnaiz Sánchez Universidad de Murcia, España

Xavier Besalú Costa Universitat de Girona, España

Jose Joaquin Brunner Universidad Diego Portales, Chile

Damián Canales Sánchez Instituto Nacional para la Evaluación de la Educación, México

María Caridad García Universidad Católica del Norte, Chile

Raimundo Cuesta Fernández IES Fray Luis de León, España

Marco Antonio Delgado Fuentes Universidad Iberoamericana, México

Inés Dussel FLACSO, Argentina

Rafael Feito Alonso Universidad Complutense de Madrid, España

Pedro Flores Crespo Universidad Iberoamericana, México

Verónica García Martínez Universidad Juárez Autónoma de Tabasco, México

Francisco F. García Pérez Universidad de Sevilla, España

Edna Luna Serrano Universidad Autónoma de Baja California, México

Alma Maldonado Departamento de Investigaciones Educativas, Centro de Investigación y de Estudios Avanzados, México

Alejandro Márquez Jiménez Instituto de Investigaciones sobre la Universidad y la Educación, UNAM México

José Felipe Martínez Fernández University of California Los Angeles, USA
Fanni Muñoz Pontificia Universidad Católica de Perú

Imanol Ordorika Instituto de Investigaciones Economicas - UNAM, México

Maria Cristina Parra Sandoval Universidad de Zulia, Venezuela

Miguel A. Pereyra Universidad de Granada, España

Monica Pini Universidad Nacional de San Martín, Argentina

Paula Razquin UNESCO, Francia

Ignacio Rivas Flores Universidad de Málaga, España

Daniel Schugurensky Arizona State University

Orlando Pulido Chaves Universidad Pedagógica Nacional, Colombia

José Gregorio Rodríguez Universidad Nacional de Colombia

Miriam Rodríguez Vargas Universidad Autónoma de Tamaulipas, México

Mario Rueda Beltrán Instituto de Investigaciones sobre la Universidad y la Educación, UNAM México

José Luis San Fabián Maroto Universidad de Oviedo, España

Yengny Marisol Silva Laya Universidad Iberoamericana, México

Aida Terrón Bañuelos Universidad de Oviedo, España

Jurjo Torres Santomé Universidad de la Coruña, España

Antoni Verger Planells University of Amsterdam, Holanda

Mario Yapu Universidad Para la Investigación Estratégica, Bolivia 